periods of chopping the degree of comminution obtained is considered to be roughly proportional to the time of chopping. Samples were withdrawn at intervals and allowed to stand. The concentration of ascorbic acid was determined in these samples after two hours, when a steady state had been reached, and again after twenty-two hours. At the same time the amount of juice which could be pressed from each sample under certain conditions was deter. mined. The results are plotted in Figs. 2 and 3 and

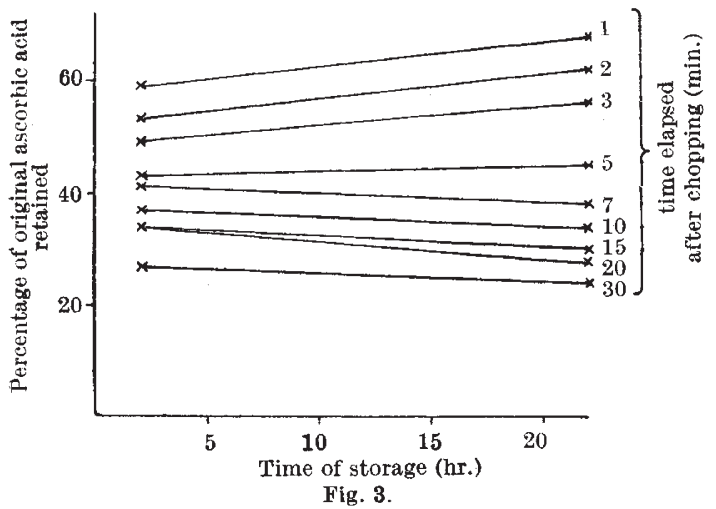

indicate that the longer the time of chopping the greater the percentage of ascorbic acid lost and the greater the amount of juice which may be obtained by pressing. In Fig. 2 the correspondence between the curves for the percentage of ascorbic acid lost and the percentage of residual material after pressing may be fortuitous.

The Lyons Laboratories,

L. H. LAMPitT.

L. C. BAKER.

T. L. Parkinson.

London, W.14. June 3.

I Nature, 149, 499 (1942).

'NATURE, 149, 271 (1942).

\section{The 'Oxygen Trough' of Expiration}

I have been unable to confirm ${ }^{1}$ the presence of an alveolar air plateau as described by Haldane ${ }^{2}$. Two of the subjects investigated gave paradoxical results in that the ultimate alveolar sample had a lower

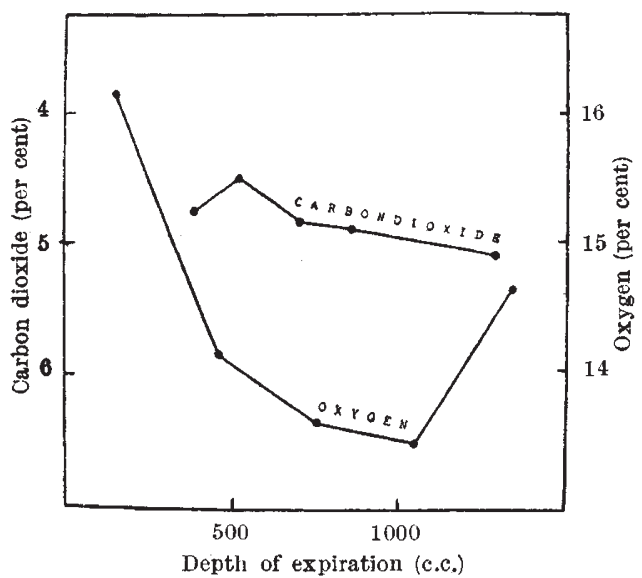

carbon dioxide tension than the penultimate. In a paper presented at the same time, Cotton ${ }^{3}$ examined the oxygen percentages, and demonstrated what he later described as the "oxygen trough of expiration". His oxygen figures were in harmony with my results; that is, the percentage of oxygen of the ultimate sample was higher than that of the penultimate.

The oxygen results from one of the subjects used by me are given in the accompanying graph. They confirm the findings of Cotton.

No satisfactory explanation of this phenomenon, which has recently been confirmed by Marenzi and Costoya ${ }^{4}$, is at present available. It seems reasonable to assume that it is due to inequality of ventilation, or inequality of the blood-flow to the different parts of the lung.

\section{Department of Physiology, \\ University of Manchester, Manchester, 13. May 4.}

${ }^{1}$ Mackay, I. F. S., J. Physiol., 98, 73 (1940).

2 Haldane, J. S., and Priestley, J. G., "Respiration", 20 (1935).

- Cotton, F. S., Austral. J. Exp. Biol., 17, 433 (1939).

- Marenzi, A. D., and Costoya, M. A., Rev. Soc. Argent. Biol., 17, 190 (1941).

\section{Crystal Dynamics of Rocksalt}

THe early X-ray experiments of James, Firth and Waller ${ }^{1}$ on the modification of the diffracting power of crystals of sodium chloride due to thermal vibrations of the atoms dealt only with the reduction in intensity of the Bragg reflexions at different tempera. tures, and could therefore only give average root mean square amplitudes, but the more recent diffuse spot methods promise to give information concerning the whole frequency spectrum. By observation of the position, shape and intensity of the diffuse spots when the crystal is turned into a succession of reflecting positions, the effect of vibrations travelling in different directions, polarized in definite ways and with frequencies within known limits, can be traced ${ }^{2,3,4}$.

The above interpretation of the diffuse spot photographs has, however, been challenged by Sir C. V. Raman $^{5}$, and one of his colleagues, Dr. C. S. Venkateswaran ${ }^{6}$, has published experimental results for sodium chloride crystals which are claimed to be inconsistent with the thermal (Faxén-Waller) theory. Since the challenge includes a denial of the validity of the Born theory of lattice dynamics, it is important that the experimental evidence should be carefully examined.

Venkateswaran's observations are as follows: (1) variation of diffuse spot intensity with temperature (one crystal setting, three temperatures); (2) comparison of diffuse spot and Laue spot intensities for three different irradiated volumes of crystal, using a slightly divergent beam; (3) measurements of relative sharpness; and (4) intensity, of 200, 400,600 reflexions (molybdenum unfiltered radiation, one crystal setting); (5) comparison of 111,222 reflexions ; (6) measurement of the 'drift' of the diffuse maxima towards the Laue spots as the crystal rotates.

Every one of these experimental observations is either inaccurately performed or wrongly interpreted. The main criticisms are as follows :

(1) Venkateswaran has neglected the falling off of 